\title{
Shape-Dependent Gloss Correction
}

\author{
Peter Vangorp* \\ Philip Dutré ${ }^{\prime}$ \\ Department of Computer Science \\ Katholieke Universiteit Leuven
}

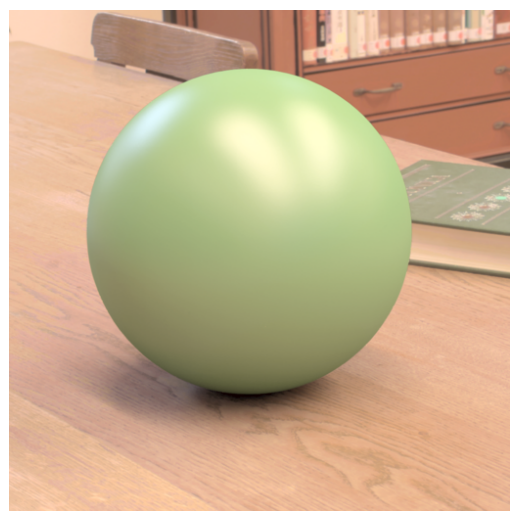

(a) Selected material

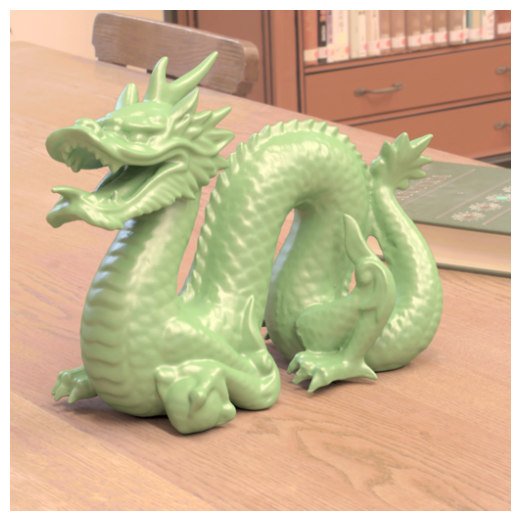

(b) Uncorrected transfer

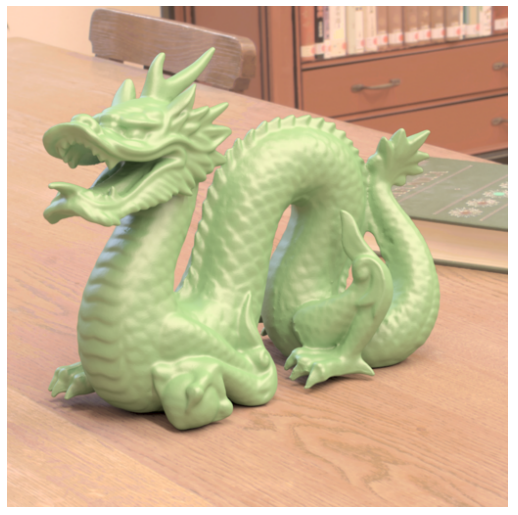

(c) Corrected transfer

Figure 1: Transferring a material from (a) a sphere to (b) a dragon results in exaggerated gloss. (c) The shape-dependent gloss correction presented in this paper preserves the appearance.

\section{Abstract}

Visual observation is one of the primary means to interact with the world around us. However, the interplay of geometry, material, and illumination in the perception of objects is still not entirely understood.

It is generally accepted that the shape of objects influences the perception of their material appearance. In this paper we investigate and quantify the influence of shape on gloss perception in a realistic setting, using a general cue combination framework and likelihood analysis. Our results show that this influence can be quantified, and that a simple additive model between the shape cue and the actual gloss cue adequately describes this influence.

This paper contributes to a deeper understanding of the perception of objects, and also enables new applications such as a material selection tool that corrects for the influence of gloss when switching between shapes. This improves the intuitiveness of material selection tools for novices as well as professional artists.

CR Categories: I.3.7 [Computer Graphics]: Three-Dimensional Graphics and Realism-Color, shading, shadowing, and texture; J.4 [Social and Behavioral Sciences]: Psychology

Keywords: material editing, gloss, shape, cue combination, maximum likelihood estimation

\footnotetext{
*e-mail: peter.vangorp@cs.kuleuven.be

†e-mail: philip.dutre@cs.kuleuven.be
}

\section{Introduction}

Computer graphics artists spend a lot of time on the difficult task of selecting materials for their 3D scenes. Most modeling applications offer only primitive material selection tools to help them. Usually the interface provides a slider for each parameter of the underlying material model. The meaning of these parameters follows from the mathematical or physical description of materials. For instance, the width of a specular lobe function is related to the physical property of surface roughness. However, people intuitively think of the appearance of materials in terms of intensity of the highlights and sharpness of the reflected image. Several of the traditional mathematical or physical parameters may influence each of these perceptual aspects in a non-linear manner, further complicating the material selection task.

Pellacini et al. [2000] designed a novel material model allowing an artist to specify the appearance with perceptually-based parameters. Additionally, each parameter was designed to describe an independent aspect of material appearance, using a perceptually linear scale. Research into the usability of material selection tools has also lead to more intuitive interfaces, including a "painting" paradigm to specify the location and shape of highlights on a surface [Colbert et al. 2006], and a novel way of navigating the space of materials by example images [Ngan et al. 2006].

A major outstanding problem in current material selection tools is transferring materials from one shape to another. Materials are often visualized on a sphere during the interactive selection process, and the mathematical parameters are then used for render-

Copyright $(\odot 2008$ by the Association for Computing Machinery, Inc.

Permission to make digital or hard copies of part or all of this work for personal or classroom use is granted without fee provided that copies are not made or distributed for commercial advantage and that copies bear this notice and the full citation on the first page. Copyrights for components of this work owned by others than ACM must be honored. Abstracting with credit is permitted. To copy otherwise, to republish, to post on servers, or to redistribute to lists, requires prior specific permission and/or a fee. Request permissions from Permissions Dept, ACM Inc., fax +1 (212) 869-0481 or e-mail permissions@acm.org.

APGV 2008, Los Angeles, California, August 9-10, 2008.

C) 2008 ACM 978-1-59593-981-4/08/0008 $\$ 5.00$ 
ing the target shape. Figure 1 demonstrates that this causes a shift in the appearance of the material, and the result does not look as the artist had intended. Recent developments in material editing try to solve this problem by visualizing materials directly on the target shape [Ben-Artzi et al. 2006; Colbert and Křivánek 2007a Sun et al. 2007; Ben-Artzi et al. 2008]. However, many of these techniques are computationally expensive and difficult to incorporate into existing modeling applications.

In this paper we present the novel idea of shape-dependent gloss correction: we undo the gloss shift caused by transferring a material to a different object. This practical solution can easily be added to any existing modeling application. In Section 3 we describe our perceptual experiments designed to measure the extent of the shift in gloss that occurs in standard uncorrected material transfer. In Section 4 the experimental results are analyzed and a simple model is found to describe the required gloss correction. In Section 5 we improve an existing material editor with gloss correction, driven by our experimental model and data.

\section{Related Work}

Perceptual material parameterization. Pellacini et al. [2000] examined the perceptual space of glossy materials representable by the isotropic Ward reflectance model [Ward 1992]. They used multidimensional scaling (MDS) to show that there are 2 perceptual gloss dimensions present in this space, namely contrast gloss and distinctness-of-image (DOI) gloss. These dimensions are used as the basis for a perceptually uniform reparameterization of the Ward reflectance model. Additionally, Ferwerda et al. [2001] derive the just noticeable differences or thresholds for perceiving differences in this perceptual gloss space.

Material constancy. Early work in visual perception has focused mainly on the physiological and neurological characteristics of the human visual system related to contrast and color. Examples include research into different aspects of color constancy: the color and lightness of an object appear remarkably constant under substantial changes in illumination (see [Brainard 2004] for a recent overview). Although limits of color constancy have been discovered [Foster 2003; Brainard and Wandell 1991], it remains one of the strongest factors in the visual perception of materials.

Similar invariance of perceived gloss under changing illumination, termed gloss constancy, was demonstrated by Obein et al. [2004]. While it is often assumed that color constancy across changing surface gloss also holds [ASTM 1999; Aida 1997], this is certainly not always the case. Xiao and Brainard [2006] demonstrated that color appearance is indeed affected slightly by gloss. Pellacini et al. [2000] designed their reparameterization to achieve gloss constancy of each independent gloss parameter across changes in the other parameter and across changes in diffuse color.

Influence of shape. Material selection tools traditionally visualize the material on a simple sphere. It shows highlights as well as diffuse color, which are the two most important perceptual cues for material recognition. However, artists often encounter situations where the material looks very different on the target object, indicating that gloss constancy is not guaranteed when varying the geometry. In [Vangorp et al. 2007] we studied the extent of the influence of shape and proved it could indeed lead to significant changes in appearance. Additionally, we found that material discrimination thresholds varied widely for different shapes.

Braje and Knill [1994] discovered that geometric frequency content is correlated to the perception of specularity. Nishida and Shinya [1998] found that observers had great difficulty matching glossy, bumpy surfaces of different amplitudes and frequencies, and they relate observers' performance to information in the luminance histogram of the stimulus images. Berzhanskaya et al. [2005] found that the impression of local gloss decreases with the distance from the highlight and with any surface discontinuities along that distance.

Ho et al. [2008] quantified the mutual influence of gloss and bumpiness: not only do bumpy surfaces look more glossy, but glossy surfaces also look more bumpy. They showed these influences could be modeled by a simple additive formula.

Influence of illumination. Another drawback of many traditional material selection tools is the use of a single point light source to illuminate the material sample. The reflectance matching experiments of Fleming et al. [2003] indicate that people can judge material characteristics more accurately under natural environment illumination than under artificial illumination like point light sources. Additionally, te Pas and Pont [2005] demonstrated that the effects of illumination, reflectance, and bumpiness are often confounded.

Ramamoorthi et al. [2001] demonstrated from a signal-processing viewpoint that the appearance of an object is a combination of geometry, material, and illumination, and that the effects of these cues are generally indistinguishable without additional assumptions. This fact was exploited by Ramanarayanan et al. [2007] to relate changes in geometry or material to equivalent changes in illumination. They constructed a measure for visual equivalence based on variations of this single cue.

Real-time material editing. Colbert et al. [2006] presented a BRDF editor based on a multi-lobe extension of the Ward reflectance model. The editor allows an artist to paint and manipulate highlights on a point-lit sphere, chosen to show clearly distinct highlights. The resulting material is at the same time rendered on a more complex model under unshadowed environment map illumination to see if the material gives the desired result on the target shape. Colbert and Křivánek [2007a; 2007b] presented a similar BRDF editor for several popular reflectance models, using advanced sampling techniques on the GPU for increased speed and minimal precomputation time.

The BRDF editor of Ben-Artzi et al. [2006; 2008] allows the user to visualize arbitrary reflectance functions directly on the target shape, in complex scenes, under fixed viewpoint and illumination. Sun et al. [2007] presented a fully dynamic BRDF editor with variable viewpoint and lighting. Both systems offer intuitive controls to select materials, and still produce high-quality shadowing and global illumination effects at interactive rates, at the expense of considerable precomputation time.

\section{Perceptual Experiments}

We have designed a set of experiments to measure the extent of the gloss shift that can occur, so that we can correct it. We focus on a limited set of realistic conditions to obtain statistically significant results that apply to real-world material editing tasks.

\subsection{Stimulus Images}

The stimulus images are augmented reality images constructed from these 3 components:

1. The scene provides a background and illumination environment, in which an object of interest is placed (Figure 2(a)). 

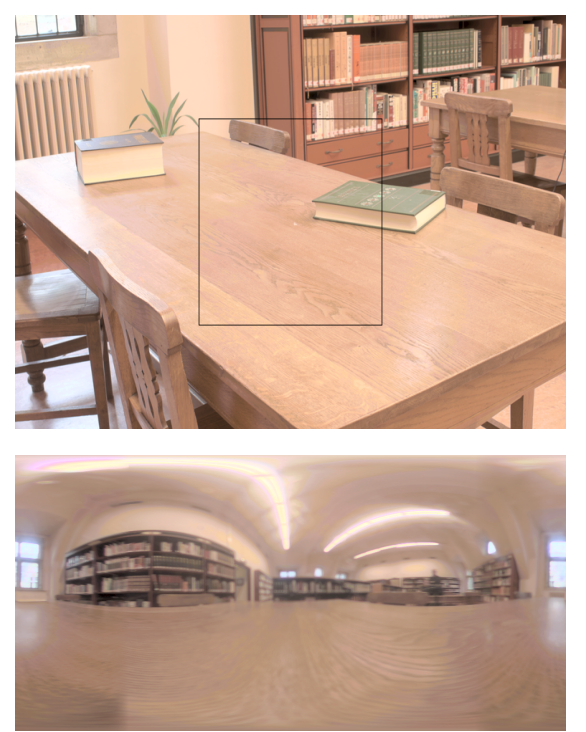

(a) Scene

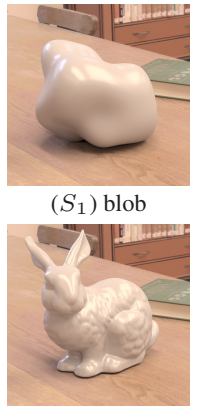

( $S_{3}$ ) bunny

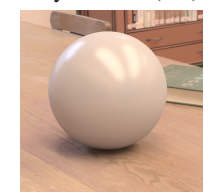

$\left(S_{5}\right)$ sphere

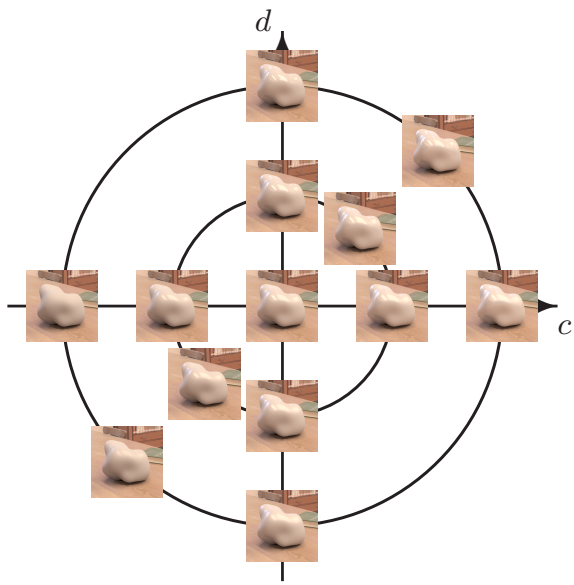

(c) Materials

Figure 2: Components of the stimulus images. (a) Background photograph and environment illumination map of the library scene. Stimulus images were cropped to the square frame. (b) The shapes used in the stimulus images. (c) The gloss variations used in the stimulus images lie on isogloss difference contours in the Euclidean $\{c, d\}$-space.

2. An object with a distinctive shape is placed in the scene (Figure 2(b)).

3. The object is rendered with a variation of a light grey plastic material (Figure 2(c)).

The objects are placed in a real-world scene using differential rendering techniques [Debevec 1998] to achieve the required level of realism. We use natural environment illumination to improve material discrimination, as suggested by Fleming et al. [2003]. All images use the same scene and illumination environment, captured in a library (Figure 2(a)). This specific illumination environment was chosen because it produces on each object a mix of frontal highlights from the ceiling lights, as well as grazing highlights from the window at the top left. We show 2 separate images in a single trial, each containing 1 object, so that the object is always in the center of the image and highlights are always in the same place on a shape.

We chose the following 5 shapes: a blob, a buddha, a bunny, a dragon, and a sphere (Figure 2(b)). Each shape is scaled to fill the image. These abstract shapes and statuettes do not have a "natural" size, so they will not look out of proportion to the background.

To fully utilize observers' experience with real-world materials, we chose a common material like plastic, represented by the isotropic Ward-Dür BRDF model [Ward 1992; Dür 2006]:

$$
f_{r}=\frac{\rho_{d}}{\pi}+\frac{\rho_{s}}{4 \pi \alpha^{2} \cos \theta_{i} \cos \theta_{o}} \exp \left(-\frac{\tan ^{2} \theta_{h}}{\alpha^{2}}\right)
$$

where $\rho_{d}$ is the diffuse reflectance, $\rho_{s}$ is the specular reflectance, $\alpha$ is a surface roughness parameter, and $\theta_{i}, \theta_{o}$, and $\theta_{h}$ are the elevation angles of the incident light direction, the outgoing light direction and the half vector, respectively. The parameters $\rho_{d}$, $\rho_{s}$, and $\alpha$ were chosen inside the plausible range for real-world plastics, based on measurements and fitted data for such materials [Matusik et al. 2003; Ngan et al. 2005]. The main advantages of the Ward-Dür model over tabulated measurement data or other analytical models include its simplicity and efficiency, and the fact that it is physically plausible within a certain parameter range.
The reparameterization by Pellacini et al. [2000] was used to generate perceptually uniform gloss variations on the base materials. The dimensions of contrast gloss $(c)$ and distinctness-of-image gloss $(d)$ are specified as independent transformations of the parameters in the BRDF model:

$$
\begin{gathered}
c=\sqrt[3]{\rho_{s}+\rho_{d} / 2}-\sqrt[3]{\rho_{d} / 2} \\
d=(1-\alpha)
\end{gathered}
$$

This reparameterization was intended for the original Ward model, but the Ward-Dür model introduces only an improved normalization which makes reflection at grazing angles more realistically plausible. From experience we know that the Ward-Dür parameter values are approximately equal to the Ward parameter values for most real-world materials [Ngan et al. 2005].

Pellacini et al. [2000] also introduced a Euclidean $\{c, d\}$-space defined by the distance metric $D_{i, j}$ between two materials $i$ and $j$ :

$$
D_{i, j}=\sqrt{\left(c_{i}-c_{j}\right)^{2}+\left(1.78\left(d_{i}-d_{j}\right)\right)^{2}}
$$

For our main experiment we select five equidistant variations of the material along the diagonal in this space, where $c$ and $d$ vary simultaneously [Ho et al. 2008]. This diagonal was chosen because people associate gloss with polishing, which generally increases both aspects of gloss. For two additional experiments we again choose five equidistant variations of the material, but along a single gloss dimension. We reuse the middle variation of the main experiment ( $c=0.0987, d=0.9145$ ) and construct the variations on either side along the $c$ or $d$ dimension, at the same perceptual distances $D_{i, j}=0.0493$. This distance is near the material discrimination threshold so it should be easy to compare non-adjacent gloss variations when presented on identical shapes, but it may be difficult when they are presented on different shapes. The diffuse reflectance of the light grey plastic material is kept constant at $\rho_{d}=0.33$. All gloss variations are displayed in Figure 2(c). 


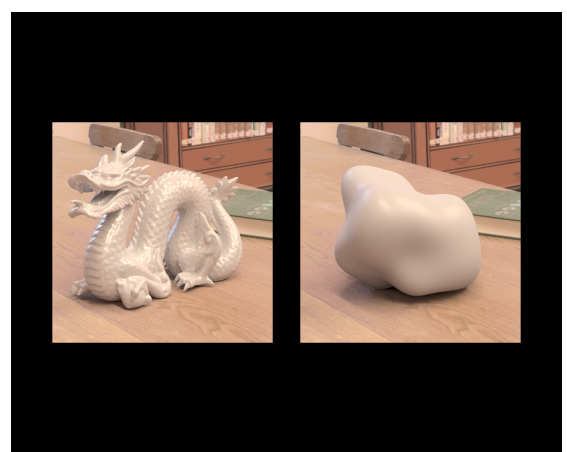

Figure 3: Example of stimulus presentation for each trial.

\subsection{Procedure}

Participants were given written instructions before the experiment, explaining the technical term "glossy" as "shiny" or "polished". For each trial a pair of stimulus images was presented side-by-side on a calibrated 19" LCD monitor (ViewSonic VP930) under controlled lighting conditions $\sqrt[\square]{\square}$ Each pair filled approximately $40 \%$ of the screen area over a black background (Figure 3). The question asked for each trial was: "Which object is more glossy?"

In a short training session of 75 image pairs, both images displayed the same shape and there could only be a difference in gloss. The gloss parameters were chosen to be easily discriminable in such a setting, and indeed participants gave the correct answer on average $97 \%$ of the time. Note that 25 pairs of identical images were included in this experiment and both answers were considered correct in these cases, so the range of possible scores is 33\%-100\%. One participant who scored less than $90 \%$ in the training experiment was classified as an outlier and their data was excluded from the analysis.

The main and additional experiments consisted of 325 image pairs of potentially different shapes and gloss parameters. Comparing the gloss of different shapes can be more difficult, as evidenced by the average scores of $92 \%, 89 \%$, and $86 \%$. These experiments also included 25 identical pairs, so the range of possible scores is $8 \%-100 \%$. Most participants completed the experiments in under 45 minutes. To avoid possible lapses in concentration, the instructions explicitly stated that participants were allowed to take a short break if they grew tired. All participants had normal or correctedto-normal visual acuity and normal color vision. All participants were unpaid volunteers.

A total number of 16 observers participated in the main experiment: 5 computer graphics researchers who knew the purpose of the experiment and 11 persons who had no particular knowledge of computer graphics techniques and who were naive to the purpose of the experiment. A subset of 7 naive and 4 experienced observers participated in the additional experiment with purely contrast gloss variations, and a subset of 5 naive and 4 experienced observers performed the additional experiment with purely distinctness-of-image gloss variations. In Figure 4 the scores of the experiments show no statistically significant differences between these two groups, confirming the earlier finding that everyday experience with materials outweighs knowledge of technical vocabulary and shading models [Vangorp et al. 2007]. Additionally, none of the participants would be statistical outliers in the other group.

\footnotetext{
${ }^{1}$ The images in this paper should ideally be viewed on a similar highquality monitor.
}

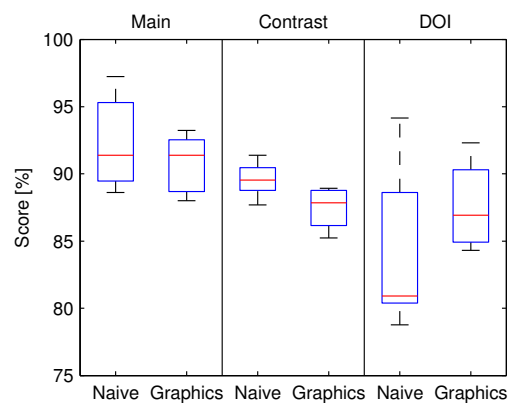

Figure 4: Box plots of the experiment scores for the two groups of participants show no significant differences.

\section{Analysis and Results}

\subsection{Cue Combination}

Perceptual phenomena are often influenced by several simultaneously available sensory cues. The processing of cues in the brain can be described as selection and weighting of the most useful cues. This model is called cue combination or fusion [Clark and Yuille 1990] and it has been applied to many perceptual topics including the visual perception of depth [Landy et al. 1995] and illumination [Maloney 2002]. The cue combination model is unaffected by the distinction between helpful cues and false cues that hinder perception, like the shape cue in our experiments.

We assume that an observer perceives the gloss of each stimulus independently, influenced by the primary cue of actual physical gloss $G$ and by the secondary cue of shape $S$. The perceived level of gloss can be described by an unknown cue combination function $f(G, S)$. We also assume that an observer forms a continuous decision variable $\Delta_{i, j}$ for the perceived difference between the gloss of two stimuli:

$$
\Delta_{i, j}=f\left(G_{i}, S_{i}\right)-f\left(G_{j}, S_{j}\right)+\epsilon, \quad \epsilon \sim N(0,1)
$$

where $\epsilon$ describes the influence of judgment errors contaminating the decision variable. We assume without loss of generality that this contamination has a standard normal distribution, and that the function $f$ is anchored to 0 for the blob $\left(S_{1}\right)$ with the lowest gloss $\left(G_{1}\right)$ :

$$
f\left(G_{1}, S_{1}\right)=0
$$

An observer decides that stimulus $i$ looks more glossy than stimulus $j$ if $\Delta_{i, j}>0$ and vice versa.

A decision matrix depicts the decision variable as the number of times that stimulus $i$ is perceived more glossy than stimulus $j$, normalized to a percentage. A negative percentage means stimulus $i$ is usually perceived less glossy than stimulus $j$. The decision matrices for each experiment, averaged over all observers, are displayed in Figure 5. They look similar to the ideal matrix, but they are clearly contaminated with judgment errors. The systematic differences in a row or column of $5 \times 5$-pixel blocks suggest the influence of shape. We assume the decision variable is unaffected by the order of stimulus presentation, so decision matrices will be approximately antisymmetric if the contamination is small:

$$
\Delta_{i, j} \approx-\Delta_{j, i}
$$

This allows us to project all values onto one half of the matrix.

We are interested in finding the simplest model for the function $f$ that will explain the experimental data sufficiently. We will consider 3 standard categories of interaction between the parameters of the function $f$ : 


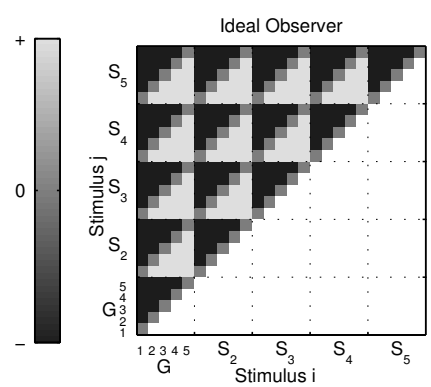

(a)

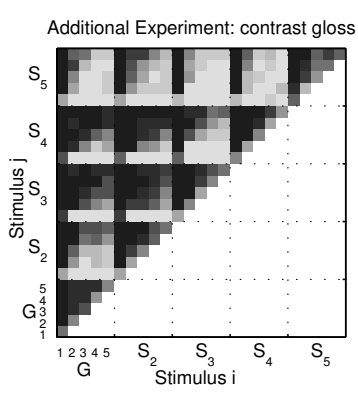

(c)

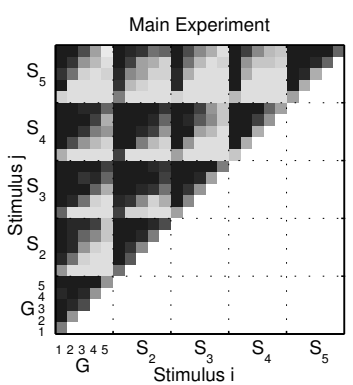

(b)

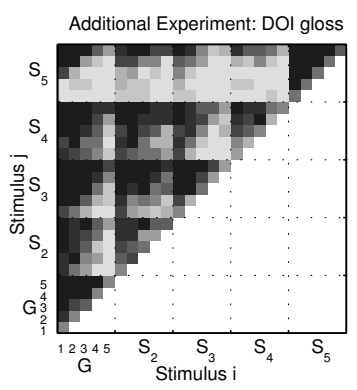

(d)
Figure 5: The decision matrices of (a) an ideal observer, and averaged over all observers for (b) the main experiment, (c) the additional contrast gloss experiment, and (d) the additional distinctness-of-image (DOI) gloss experiment. Each block of $5 \times 5$ pixels represents the shape cues of a stimulus pair. Each pixel within such a block represents the gloss cues of the stimulus pair.

Full: Gloss perception is defined by the arbitrary interaction or strong fusion of actual gloss $G$ and shape $S$ :

$$
f(G, S)=f_{G, S}(G, S)
$$

Additive: Gloss perception is defined by the additive interaction or weak fusion of actual gloss $G$ and shape $S$ :

$$
f(G, S)=f_{G}(G)+f_{S}(S)
$$

Independent: Gloss perception is not influenced by the shape cue:

$$
f(G, S)=f_{G}(G)
$$

For each of these categories we differentiate between linear and non-linear functions for the gloss component $f_{G}$, resulting in 6 different models. We used a perceptually uniform gloss scale so linearity is a reasonable assumption. The non-linear alternative does not impose any constraints on the gloss component $f_{G}$, not even monotonicity. The shapes form only a nominal scale without a meaningful ordering, so the shape component $f_{S}$ is also unconstrained and only defined on these discrete instances.

\subsection{Maximum Likelihood Estimation}

The 6 different models are fitted to the experimental data using the Maximum Likelihood Estimation method [Eliason 1993]. Table 1 shows the required number of parameters or degrees of freedom for each model, and the actual parameters we estimate directly. Values at other stimuli can be derived from these parameters using the constraints of each model.

\begin{tabular}{l|r|l} 
Model & DoF & Parameters \\
\hline \hline Non-linear, full & 24 & $f_{G, S}\left(G_{*}, S_{*}\right)$ \\
Linear, full & 9 & $f_{G, S}\left(G_{1}, S_{*}\right), f_{G, S}\left(G_{5}, S_{*}\right)$ \\
Non-linear, additive & 8 & $f_{G}\left(G_{*}, S_{1}\right), f_{S}\left(G_{1}, S_{*}\right)$ \\
Linear, additive & 5 & $f_{G}\left(G_{5}, S_{1}\right), f_{S}\left(G_{1}, S_{*}\right)$ \\
Non-linear, independent & 4 & $f_{G}\left(G_{*}, S_{1}\right)$ \\
Linear, independent & 1 & $f_{G}\left(G_{5}, S_{1}\right)$
\end{tabular}

Table 1: Degrees of freedom $(D o F)$ and parameters that are directly estimated. The symbol $G_{*}$ means parameters must be estimated for all 5 gloss cues. Note that the reference point $f\left(G_{1}, S_{1}\right)=0$ should be excluded from the parameter lists.

Figure 6 displays the resulting models for the complete dataset of the main experiment with 16 participants. The non-linear models display a nearly linear relation in the higher gloss range, but exhibit a bend at the low end. The dependent models show that there is clearly a difference in gloss perception between the shapes. The curves of the full model are approximately parallel, suggesting the additive model is sufficient to describe the influence of shape on gloss perception. An additive relation means that the perceptual gloss scales are parallel, the effect of each shape is described by a single offset, and an ordering exists between shapes.

In Figure 7 we show the non-linear and linear additive models for the complete datasets of the additional experiments. The distinctness-of-image gloss scale looks strikingly linear, but the contrast gloss scale again exhibits a non-linearity in the lower gloss range. This non-linearity might be inherent in the contrast gloss parameterization or it might be an artifact caused by using this parameterization in conjunction with the Ward-Dür reflectance model.

\subsection{Likelihood Ratio tests}

To prove the statistical significance of the trends observed in Figures 6-7 we use the Likelihood Ratio test. It is a statistical hypothesis test that chooses between the null hypothesis $H_{0}$ that two models $i$ and $j$ explain the experimental data equally well, and the alternate hypothesis $H_{A}$ that one model explains the data significantly better than the other. The $\log$-likelihoods $\log L$ returned by the Maximum Likelihood Estimation, which indicate the goodnessof-fit of the models, are compared using the test statistic $x_{i, j}$ :

$$
x_{i, j}=2\left(\log L_{i}-\log L_{j}\right)
$$

For large samples, the test statistic $x_{i, j}$ has a $\chi^{2}$ distribution with degrees of freedom equal to the difference in the number of parameters in the models.

Tables 2-3 show a representative subset of the Likelihood Ratio tests for each experiment, confirming the statistical significance of our earlier observations. In general, we can conclude that the nonlinear, additive model is sufficient to predict the influence of shape on gloss perception.

\section{Material Editing with Gloss Correction}

Current material editors switch between shapes without updating the gloss parameters, keeping constant physical gloss. We extended the material editor of Colbert and Krrivánek [2007a] to update the gloss parameters between shapes, preserving perceptual gloss.

The contrast gloss and distinctness-of-image gloss dimensions represent orthogonal axes in the perceptual gloss space. We can update both parameters by moving along the diagonal of the main experiment, or we can update only a single gloss parameter using the data from the additional experiments. The magnitude of the correction 

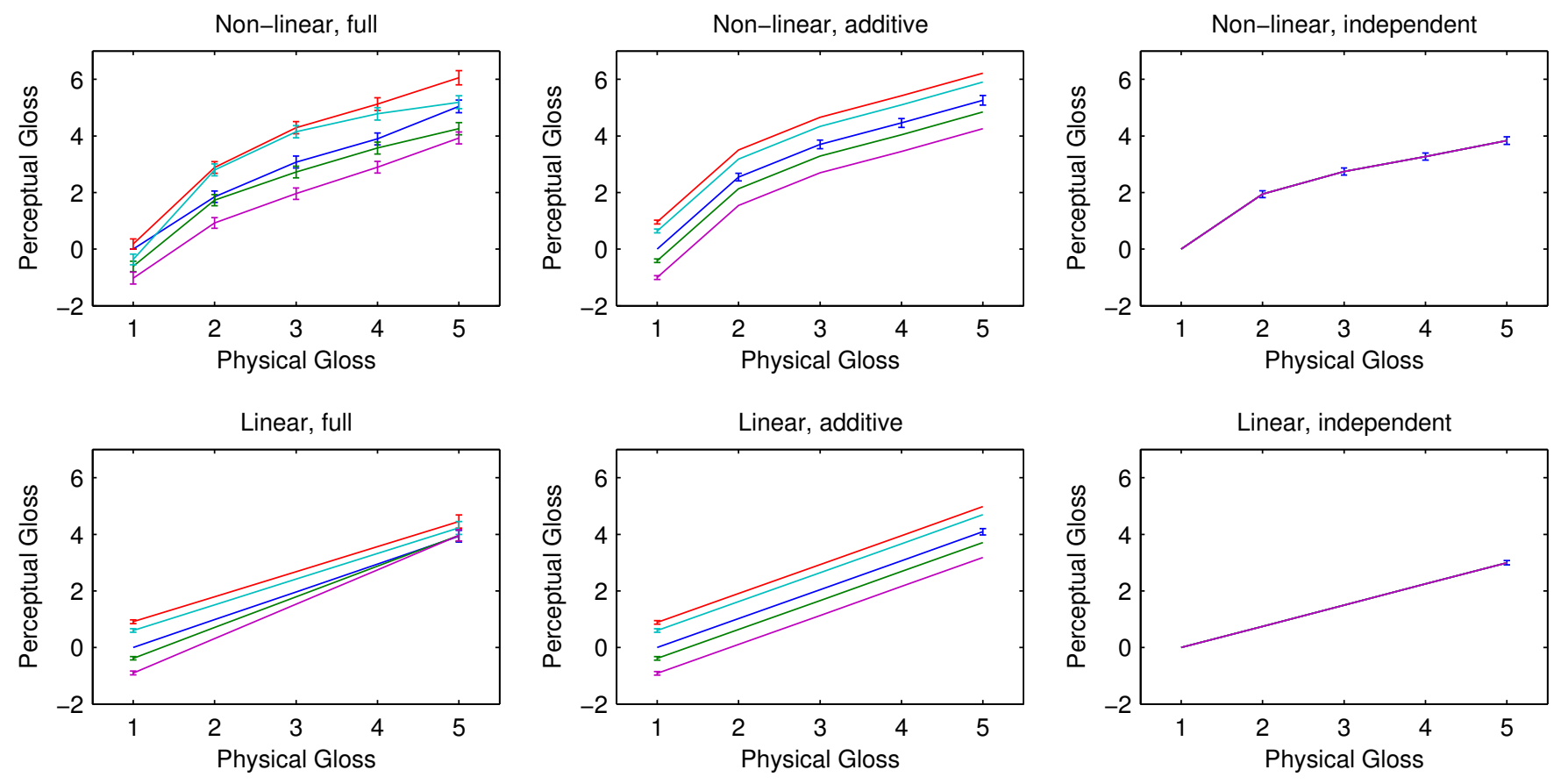

Legend: $\quad$ blob

buddha bunny _ dragon

sphere

Figure 6: Perceptual gloss scales returned by Maximum Likelihood Estimation for the main experiment, averaged over all $N=16$ observers. Error bars show the standard error for the points that were directly estimated for each model. The blob at physical gloss level 1 is always anchored to perceptual gloss level 0 .
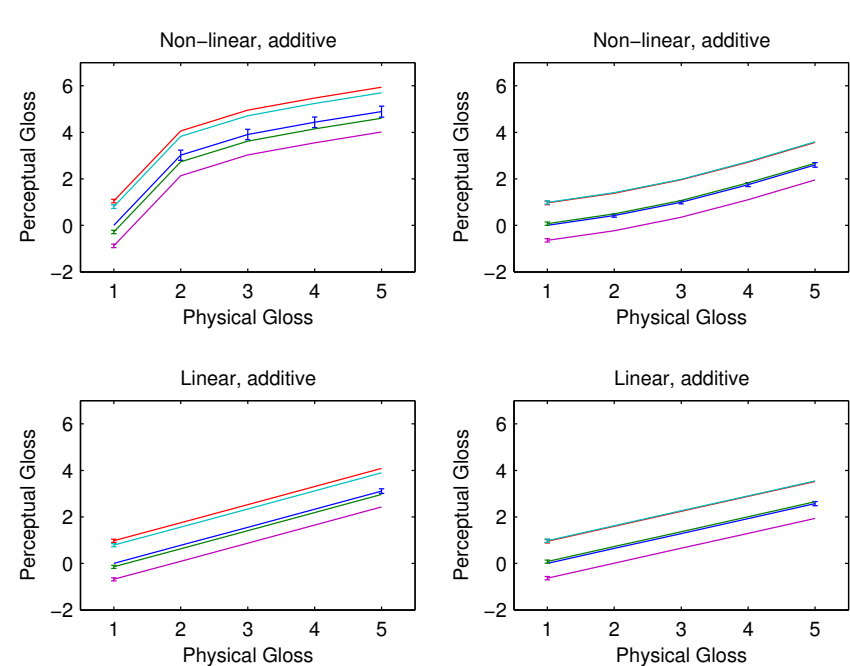

(a) Contrast gloss

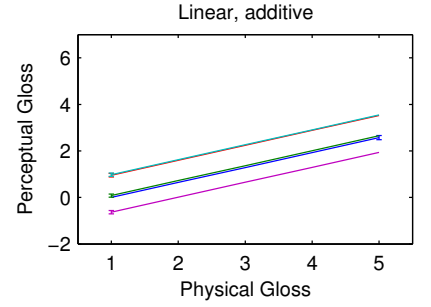

(b) Distinctness-of-image gloss

- blob — buddha — bunny — dragon - sphere

Figure 7: Additive perceptual gloss scales returned by Maximum Likelihood Estimation for the additional experiments. (a) Contrast gloss experiment, averaged over all $N=11$ observers. (b) Distinctness-of-image gloss experiment, averaged over all $N=$ 9 observers.

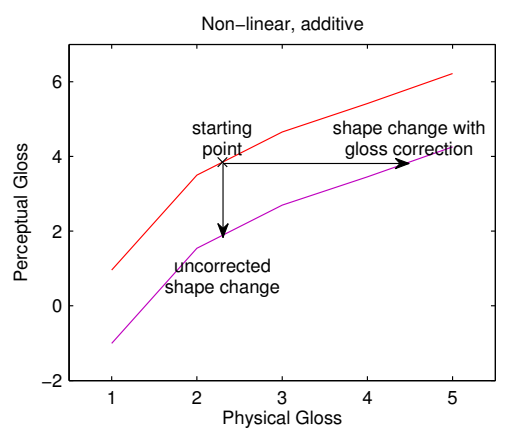

Figure 8: Gloss correction keeps perceptual gloss at a constant level across different shapes.

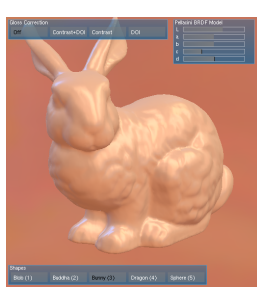

(a) Starting point

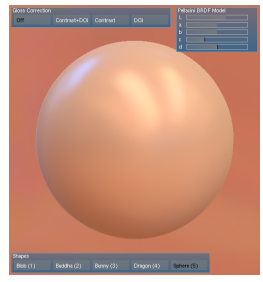

(b) Uncorrected

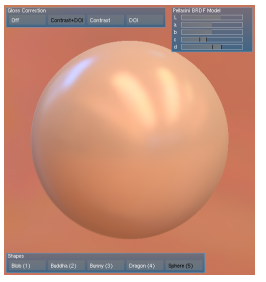

(c) Corrected
Figure 9: Screenshots of the material editor demonstrating the gloss correction along the diagonal of the gloss space. 


\begin{tabular}{|c|c|c|c|c|c|}
\hline Example: & Full & & dditive & & Independent \\
\hline Non-linear & $\underset{\uparrow p}{\log L}$ & $\stackrel{p}{\longrightarrow}$ & $\log _{\uparrow p} L$ & $\stackrel{p}{\longrightarrow}$ & $\log _{\uparrow p} L$ \\
\hline Linear & $\log L$ & $\stackrel{p}{\longleftrightarrow}$ & $\log L$ & $\stackrel{p}{\longleftrightarrow}$ & $\log L$ \\
\hline \multirow[t]{2}{*}{ JS } & $\begin{array}{r}-40.9 \\
\lceil 0.000\end{array}$ & $\stackrel{0.065}{\longleftrightarrow}$ & $\begin{array}{r}-53.6 \\
\uparrow 0.000\end{array}$ & $\stackrel{0.000}{\longleftrightarrow}$ & $\begin{array}{r}-110.0 \\
\lceil 0.000\end{array}$ \\
\hline & -68.9 & $\stackrel{0.930}{\longleftrightarrow}$ & -69.3 & $\stackrel{0.000}{\longleftrightarrow}$ & -119.4 \\
\hline \multirow[t]{2}{*}{ KD } & $\begin{array}{r}-69.2 \\
\downarrow 0.000\end{array}$ & $\stackrel{0.061}{\longleftrightarrow}$ & $\begin{array}{r}-82.0 \\
\uparrow 0.000\end{array}$ & $\stackrel{0.000}{\longleftrightarrow}$ & $\begin{array}{r}-117.8 \\
\uparrow 0.000\end{array}$ \\
\hline & -96.2 & $\stackrel{0.350}{\longleftrightarrow}$ & -98.4 & $\stackrel{0.000}{\longleftrightarrow}$ & -130.5 \\
\hline \multirow[t]{2}{*}{$\mathbf{A} \mathbf{L}^{*}$} & $\begin{array}{r}-80.0 \\
\uparrow 0.026\end{array}$ & $\stackrel{0.228}{\longleftrightarrow}$ & $\begin{array}{r}-89.9 \\
\uparrow 0.001\end{array}$ & $\stackrel{0.000}{\longleftrightarrow}$ & $\begin{array}{r}-123.6 \\
\uparrow_{0.002}\end{array}$ \\
\hline & -93.7 & $\stackrel{0.052}{\longleftrightarrow}$ & -98.4 & $\stackrel{0.000}{\longleftrightarrow}$ & -131.1 \\
\hline \multirow[t]{2}{*}{$\mathbf{T L}^{*}$} & $\begin{array}{r}-47.0 \\
\uparrow 0.000\end{array}$ & $\stackrel{0.020}{\longleftrightarrow}$ & $\begin{array}{r}-61.8 \\
\uparrow 0.000\end{array}$ & $\stackrel{0.000}{\longleftrightarrow}$ & $\begin{array}{r}-135.9 \\
\uparrow 0.000\end{array}$ \\
\hline & -81.3 & $\stackrel{0.940}{\longleftrightarrow}$ & -81.6 & $\stackrel{0.000}{\longleftrightarrow}$ & -146.6 \\
\hline
\end{tabular}

Table 2: Log-likelihoods and p-values of Likelihood Ratio tests for the results of the main experiment for $N=4$ representative observers (experienced observers indicated with ${ }^{*}$ ). The Bonferronicorrected significance level $\alpha=0.01$.

is calculated using the non-linear, additive models for each of our experiments, as shown in Figure 8. Figure 9 shows screenshots of an interactive material editing session.

Our experience with the material editor indicates that the experimental data remain valid despite the absence of shadows, interreflections, and the background photograph. The perceptual gloss scales were also designed to be independent of the diffuse color, as shown in Figure 1. We speculate that the data also remains valid for small perturbations of the viewpoint or the dominant illumination direction. Large perturbations can have a large influence on the highlights and may even change the relative ordering of the gloss perception curves, resulting in corrections that increase the gloss shift.

\section{Conclusion and Future Work}

We have introduced the novel aspect of shape-dependent gloss correction in material selection tools. We provide a practical way to calculate the parameter adjustments required to preserve perceptual gloss between different shapes. This solves a prevalent problem faced even by experienced artists, and makes the process of material selection much more intuitive. We plan to conduct a formal user study to assess the productivity gain.

The current system is limited to a fixed set of shapes and viewpoints. However, the influence of shape proves to be an additive effect which can be described by a single offset for each shape. Possibly a procedure could be developed for calculating that offset directly from a geometric description of the shape, removing the need to repeat the experiments for additional shapes.

\section{Acknowledgments}

We thank all the people who volunteered to participate in our experiments. We thank Yun-Xian Ho for helping with the implementa- tion of the likelihood functions. We would also like to acknowledge Mark Colbert and Jaroslav Křivánek for releasing their material editor, and the Stanford 3D Scanning Repository for providing the buddha, bunny, and dragon models.

\section{References}

AIDA, T. 1997. Glossiness of colored papers and its application to specular glossiness measuring instruments. Systems and Computers in Japan 28, 1, 95-112.

ASTM. 1999. Annual Book of ASTM Standards, volume 06.01. American Society for Testing and Materials.

Ben-Artzi, A., Overbeck, R., AND RAmamoorthi, R. 2006. Real-time BRDF editing in complex lighting. ACM Transactions on Graphics 25, 3, 945-954.

Ben-Artzi, A., EgAn, K., RAmAmoorthi, R., AND DuRAND, F. 2008. A precomputed polynomial representation for interactive BRDF editing with global illumination. ACM Transactions on Graphics 27, 2, 13:1-13:13.

Berzhanskaya, J., Swaminathan, G., Beck, J., And MinGOLLA, E. 2005. Remote effects of highlights on gloss perception. Perception 34, 5, 565-575.

BRAinard, D. H., AND WANDEll, B. A. 1991. A bilinear model of the illuminant's effect on color appearance. In Computational Models of Visual Processing, M. S. Landy and J. A. Movshon, Eds. MIT Press, 171-186.

Brainard, D. H. 2004. Color constancy. In The Visual Neurosciences, L. M. Chalupa and J. S. Werner, Eds. MIT Press, 948-961.

BraJe, W. L., AND KNILl, D. C. 1994. Apparent surface shape influences perceived specular reflectance of curved surfaces. Investigative Ophthalmology and Visual Science Supplement 35, 4, 1628.

Clark, J. J., AND Yuille, A. L. 1990. Data fusion for sensory information processing systems. Kluwer.

Colbert, M., AND KŘIVÁNEK, J. 2007. GPU-based importance sampling. In GPU Gems 3, H. Nguyen, Ed. Addison-Wesley, 459-476.

COlbert, M., AND KŘIVÁneK, J. 2007. Real-time shading with filtered importance sampling. In ACM SIGGRAPH 2007 Sketches, ACM, 71.

Colbert, M., PATtanaik, S., AND KŘIVÁneK, J. 2006. BRDFShop: Creating physically correct bidirectional reflectance distribution functions. IEEE Computer Graphics and Applications $26,1,30-36$.

Debevec, P. E. 1998. Rendering synthetic objects into real scenes: Bridging traditional and image-based graphics with global illumination and high dynamic range photography. In Proceedings of ACM SIGGRAPH 98, ACM Press / ACM SIGGRAPH, New York, M. F. Cohen, Ed., Computer Graphics Proceedings, Annual Conference Series, ACM, 189-198.

DÜR, A. 2006. An improved normalization for the Ward reflectance model. Journal of Graphics Tools 11, 1, 51-59.

Eliason, S. R. 1993. Maximum Likelihood Estimation: Logic and Practice. SAGE. 


\begin{tabular}{|c|c|c|c|c|c|}
\hline \multicolumn{6}{|c|}{ Additional Experiment: contrast gloss } \\
\hline \multirow[t]{2}{*}{$\mathbf{J P}$} & $\begin{array}{r}-60.5 \\
\uparrow 0.000\end{array}$ & $\stackrel{0.120}{\longleftrightarrow}$ & $\begin{array}{r}-71.9 \\
\uparrow 0.000\end{array}$ & $\stackrel{0.000}{\longleftrightarrow}$ & $\begin{array}{r}-130.9 \\
\uparrow 0.000\end{array}$ \\
\hline & -93.8 & $\stackrel{0.855}{\longleftrightarrow}$ & -94.5 & $\stackrel{0.000}{\longleftrightarrow}$ & -145.9 \\
\hline \multirow[t]{2}{*}{ KY } & $\begin{array}{r}-46.2 \\
\uparrow 0.000\end{array}$ & $\stackrel{0.251}{\longleftrightarrow}$ & $\begin{array}{r}-55.9 \\
\uparrow 0.000\end{array}$ & $\stackrel{0.000}{\longleftrightarrow}$ & $\begin{array}{r}-132.5 \\
\uparrow 0.000\end{array}$ \\
\hline & -83.8 & $\stackrel{0.383}{\longleftrightarrow}$ & -85.9 & $\stackrel{0.000}{\longleftrightarrow}$ & -148.8 \\
\hline \multirow[t]{2}{*}{$\mathbf{J L}^{*}$} & $\begin{array}{l}-51.1 \\
\lceil 0.000\end{array}$ & $\stackrel{0.000}{\longleftrightarrow}$ & $\begin{array}{l}-76.4 \\
\lceil 0.000\end{array}$ & $\stackrel{0.000}{\longleftrightarrow}$ & $\begin{array}{r}-143.5 \\
\lceil 0.000\end{array}$ \\
\hline & -103.2 & $\stackrel{0.019}{\longleftrightarrow}$ & -109.1 & $\stackrel{0.000}{\longleftrightarrow}$ & -164.9 \\
\hline \multirow[t]{2}{*}{$\mathbf{M S}^{*}$} & -82.4 & $\stackrel{0.004}{\longleftrightarrow}$ & -99.9 & $\stackrel{0.000}{\longleftrightarrow}$ & -134.0 \\
\hline & -119.0 & $\stackrel{0.891}{\longleftrightarrow}$ & -119.5 & $\stackrel{0.000}{\longleftrightarrow}$ & -149.9 \\
\hline
\end{tabular}

\begin{tabular}{|c|c|c|c|c|c|}
\hline \multirow[t]{2}{*}{ JW } & $\begin{array}{r}-54.3 \\
\uparrow 0.000\end{array}$ & $\stackrel{0.000}{\longleftrightarrow}$ & $\begin{array}{l}-78.0 \\
\lceil 0.145\end{array}$ & $\stackrel{0.000}{\longleftrightarrow}$ & $\begin{array}{r}-106.4 \\
\uparrow 0.180\end{array}$ \\
\hline & -79.1 & $\stackrel{0.498}{\longleftrightarrow}$ & -80.7 & $\stackrel{0.000}{\longleftrightarrow}$ & -108.9 \\
\hline \multirow[t]{2}{*}{ RG } & $\begin{array}{r}-120.3 \\
\lceil 0.054\end{array}$ & $\stackrel{0.163}{\longleftrightarrow}$ & $\begin{array}{r}-131.0 \\
\uparrow 0.056\end{array}$ & $\stackrel{0.000}{\longleftrightarrow}$ & $\begin{array}{r}-182.8 \\
\lceil 0.167\end{array}$ \\
\hline & -132.7 & $\stackrel{0.367}{\longleftrightarrow}$ & -134.8 & $\stackrel{0.000}{\longleftrightarrow}$ & -185.3 \\
\hline \multirow[t]{2}{*}{$\mathbf{P V}^{*}$} & $\begin{array}{r}-59.7 \\
\uparrow 0.002\end{array}$ & & $\begin{array}{r}-76.4 \\
\uparrow 0.325\end{array}$ & $\stackrel{0.000}{\longrightarrow}$ & $\begin{array}{r}-122.9 \\
\uparrow_{0.414}\end{array}$ \\
\hline & -77.5 & $\stackrel{0.860}{\longleftrightarrow}$ & -78.1 & $\stackrel{0.000}{\longleftrightarrow}$ & -124.4 \\
\hline \multirow[t]{2}{*}{$\mathbf{T L}^{*}$} & $\begin{array}{r}-55.6 \\
\uparrow 0.000\end{array}$ & $\stackrel{0.190}{\longleftrightarrow}$ & $\begin{array}{r}-65.9 \\
\uparrow 0.000\end{array}$ & $\stackrel{0.000}{\longleftrightarrow}$ & $\begin{array}{r}-145.1 \\
\uparrow 0.004\end{array}$ \\
\hline & -79.8 & $\stackrel{0.931}{\longleftrightarrow}$ & -80.2 & $\stackrel{0.000}{\longleftrightarrow}$ & -151.9 \\
\hline
\end{tabular}

Table 3: Log-likelihoods and p-values of Likelihood Ratio tests for the results of the additional experiments for $N=4$ representative observers (experienced observers indicated with ${ }^{*}$ ). The Bonferroni-corrected significance level $\alpha=0.01$.

Ferwerda, J. A., Pellacini, F., And Greenberg, D. P. 2001. A psychophysically-based model of surface gloss perception. In Proceedings of SPIE Human Vision and Electronic Imaging, SPIE, 291-301.

Fleming, R. W., Dror, R. O., And Adelson, E. H. 2003. Real-world illumination and the perception of surface reflectance properties. Journal of Vision 3, 5, 347-368.

Foster, D. H. 2003. Does colour constancy exist? Trends in Cognitive Sciences 7, 10, 439-443.

Ho, Y.-X., LAndy, M. S., And Maloney, L. T. 2008. Conjoint measurement of gloss and surface texture. Psychological Science 19, 2, 196-204.

Landy, M. S., Maloney, L. T., Johnston, E. B., And YounG, M. 1995. Measurement and modeling of depth cue combination: in defense of weak fusion. Vision Research 35, 3, 389-412.

MALONEY, L. T. 2002. Illuminant estimation as cue combination. Journal of Vision 2, 6, 493-504.

Matusik, W., Pfister, H., Brand, M., And McMillan, L. 2003. Efficient isotropic BRDF measurement. In Proceedings of the 14th Eurographics Symposium on Rendering, P. Dutré, F. Suykens, P. H. Christensen, and D. Cohen-Or, Eds., Eurographics, 241-248.

NGan, A., Durand, F., And Matusik, W. 2005. Experimental analysis of BRDF models. In Proceedings of the 16th Eurographics Symposium on Rendering 2005, K. Bala and P. Dutré, Eds., Eurographics, 117-126.

Ngan, A., Durand, F., And Matusik, W. 2006. Image-driven navigation of analytical BRDF models. In Proceedings of the 17th Eurographics Symposium on Rendering, T. Akenine-Möller and W. Heidrich, Eds., Eurographics, 399-407.

NishidA, S., AND SHINYA, M. 1998. Use of image-based information in judgments of surface-reflectance properties. Journal of the Optical Society of America A: Optics, Image Science \& Vision 15, 12, 2951-2965.
Obein, G., Knoblauch, K., And ViÉnot, F. 2004. Difference scaling of gloss: Nonlinearity, binocularity, and constancy. Journal of Vision 4, 9, 711-720.

Pellacini, F., Ferwerda, J. A., And Greenberg, D. P. 2000. Toward a psychophysically-based light reflection model for image synthesis. In Proceedings of ACM SIGGRAPH 2000, ACM Press / ACM SIGGRAPH, New York, K. Akeley, Ed., Computer Graphics Proceedings, Annual Conference Series, ACM, 55-64.

Ramamoorthi, R., And Hanrahan, P. 2001. A signalprocessing framework for inverse rendering. In Proceedings of ACM SIGGRAPH 2001, ACM Press / ACM SIGGRAPH, New York, E. Fiume, Ed., Computer Graphics Proceedings, Annual Conference Series, ACM, 117-128.

Ramanarayanan, G., Ferwerda, J., Walter, B., And BALA, K. 2007. Visual equivalence: towards a new standard for image fidelity. ACM Transactions on Graphics 26, 3, 76:1$76: 11$

Sun, X., Zhou, K., Chen, Y., Lin, S., Shi, J., And Guo, B. 2007. Interactive relighting with dynamic BRDFs. ACM Transactions on Graphics 26, 3, 27:1-27:10.

TE PAS, S. F., AND Pont, S. C. 2005. A comparison of material and illumination discrimination performance for real rough, real smooth and computer generated smooth spheres. In Symposium on Applied Perception in Graphics and Visualisation 2005, ACM, 75-81.

VAngorp, P., LAuriussen, J., And Dutré, P. 2007. The influence of shape on the perception of material reflectance. ACM Transactions on Graphics 26, 3, 77:1-77:9.

WARD, G. J. 1992. Measuring and modeling anisotropic reflection. Computer Graphics (Proceedings of ACM SIGGRAPH 92) 26, 2, 265-272.

XiaO, B., And Brainard, D. H. 2006. Color perception of 3D objects: Constancy with respect to variation of surface gloss. In Symposium on Applied Perception in Graphics and Visualisation 2006, ACM, 63-68. 